\title{
Higher Operating Temperature IR Detectors of the MOCVD Grown HgCdTe Heterostructures
}

\author{
P. MADEJCZYK (1), ${ }^{1,3}$ W. GAWRON,${ }^{2}$ A. KEBŁOWSKI,${ }^{2}$ K. MLYNARCZYK, ${ }^{2}$ \\ D. STEPIEN,${ }^{2}$ P. MARTYNIUK, ${ }^{1}$ A. ROGALSKI, ${ }^{1}$ J. RUTKOWSKI, ${ }^{1}$ \\ and J. PIOTROWSKI ${ }^{2}$
}

1.-Institute of Applied Physics, Military University of Technology, Kaliskiego 2, 00-908 Warsaw, Poland. 2.-Vigo System S.A., 129/133 Poznanska Str, 05-850 Ozarow Mazowiecki, Poland. 3.—e-mail: pawel.madejczyk@wat.edu.pl

This paper summarizes progress in metal organic chemical vapour deposition (MOCVD) technology achieved in recent years at the Institute of Applied Physics, Military University of Technology and VIGO System S.A. MOCVD with a wide range of composition and donor/acceptor doping and without postgrowth annealing is a very convenient tool for the deposition of $\mathrm{HgCdTe}$ epilayers used for uncooled infrared detectors. Particular attention is focused on: surface morphology improvement, doping issues, diffusion processes during growth study, substrate issues, crystallographic orientation selection. In this respect, MOCVD technology improvement influencing IR detector parameters is shown. CdTe buffer layer deposition allows $\mathrm{HgCdTe}$ heterostructure growth on GaAs substrates. Theoretical modelling using APSYS platform supports designing and better understanding of the carrier transport mechanism in detector's structures. Secondary ion mass spectrometry profiles allows to compare projected and obtained structures and revealed diffusion processes of the elements. A wide range of different types of infrared detectors operating at high operating temperature conditions has been constructed: photoresistors, non-equilibrium photodiodes, dual-band photodiodes, barrier and multiple detectors. The methodical research efforts contributed to the time constant reduction are important in many detector applications. Future challenges and prospects are also discussed.

Key words: MOCVD, HgCdTe growth, heterostructure, doping, infrared detectors

\section{INTRODUCTION}

Because infrared (IR) radiation is common in nature and a wide range of IR detector applications in almost all spheres of human activity, there is constant development of science and technology on IR sensing. The progress in IR detector technology has been associated mainly with semiconductor IR detectors, which are included in the group of photon

(Received January 8, 2020; accepted July 29, 2020; published online August 24, 2020) detectors. They are characterized both by high signal-to-noise ratio and fast response. ${ }^{1}$

The absorbed photon energy caused by incident IR radiation is comparable to thermal energies of atoms in a detector. The statistical nature of thermal generation of charge carriers is a source of noise which is reduced most commonly by cooling often to a temperature of $77 \mathrm{~K}$. Cryogenic cooling was always a serious obstacle to widespread applications of IR detectors and increasing their operating temperature without detectivity deterioration has become a major challenge for many research laboratories. ${ }^{2}$ Various ways to reduce thermal 
generation have been proposed ${ }^{3}$ for high operating temperature (HOT) conditions. Significant improvements have been obtained by the reduction of the absorber volume using optical immersion, double or multiple pass of IR radiation, ${ }^{2}$ suppression of Auger thermal generation in non-equlibrium devices, ${ }^{4}$ magnetoconcentration effect detectors, ${ }^{5}$ and recent barrier detectors. ${ }^{6}$ The non-equilibrium devices require significant bias currents and exhibit excessive low frequency noise that extends up to $\mathrm{MHz}$ range. The noise, which reduces the detectivity, as well as the high current requirements, limits widespread applications.

In spite of other competitive technologies and materials, the mercury cadmium telluride $\left(\mathrm{Hg}_{1-}\right.$ ${ }_{x} \mathrm{Cd} \mathrm{Te}$ ) is still the main material for the middle wavelength infrared (MWIR: 3-5 $\mu \mathrm{m}$ ), as well as the long wavelength infrared (LWIR: 8-12 $\mu \mathrm{m}$ ) detectors. The development of advanced epitaxial techniques such as molecular beam epitaxy $(\mathrm{MBE})^{7}$ and metal organic chemical vapour deposition (MOCVD) ${ }^{8}$ allows for the construction of complex HgCdTe heterostructure-based IR detectors for use in HOT conditions.

\section{MOCVD GROWTH TECHNIQUE}

$\mathrm{HgCdTe}$ epitaxial growth was carried out in the horizontal reactor of an AIX-200 Aixtron MOCVD unit. The system operates in the laminar flow regime with process pressures from 50 mbar to 1000 mbar using a butterfly valve for pressure control. Reactor pressure of 500 mbar was used for all successful growth runs. Hydrogen is used as a carrier gas. Dimethylcadmium (DMCd) and diisopropyl telluride (DIPTe) are used as precursors. Ethyl iodine (EI) is used as a donor and TDMAAs as an acceptor of dopant sources. DMCd and EI are delivered through the one channel while DIPTe and TDMAAs are delivered through another channel over the quartz container where elementary mercury is held. The Aixtron's gas foil rotation was applied for better composition uniformity. There are two temperature zones in the reactor: the mercury source zone and the growth zone with graphite susceptor. High-temperature annealing was used before each growth run for reactor cleaning. A gas delivery system was additionally equipped with Piezocon ultrasonic precursor concentration monitors. The usage of the Piezocon monitors contributed to a better repeatability of the growth processes. Adaptation of an EpiEye reflectometer allows for in situ monitoring.

The growth was carried out on 2-inch, epi-ready, semi-insulating (100) GaAs substrates, oriented $2^{\circ}$ toward the nearest $\langle 110\rangle$. Typically, a $3-4-\mu \mathrm{m}$ thick CdTe layer is used as a buffer layer reducing stress caused by crystal lattice misfit between GaAs substrate and $\mathrm{HgCdTe}$ epitaxial layer structure. ${ }^{9}$ The buffer also prevents gallium diffusion from the substrate to the $\mathrm{HgCdTe}$ layer. The interdiffused multilayer process (IMP) technique was applied for the HgCdTe deposition. ${ }^{10} \mathrm{HgCdTe}$ were grown at $350^{\circ} \mathrm{C}$ with the mercury source kept at temperatures in the range of $160-220^{\circ} \mathrm{C}$. The II/VI mole ratio was kept in the range from 1.5 to 5 during CdTe cycles of the IMP process. Acceptor and donor doping has been examined over a wide range of compositions and doping levels of $5 \times 10^{14} \mathrm{~cm}^{-3}$ to $5 \times 10^{17} \mathrm{~cm}^{-3}$ have been obtained. In order to reduce the mercury vacancy concentration, the $\mathrm{HgCdTe}$ heterostructures are often annealed in the reactor just after the growth (in situ) for $1 \mathrm{~h}$ in mercury-rich conditions. The obtained heterostructures were not annealed ex situ, however. ${ }^{11}$

The crystalline quality of the $\mathrm{HgCdTe}$ films depends on the MOCVD system, the substrate, the nucleation and the growth conditions. The growth of the HgCdTe epilayers with (111)B orientations has some advantages such as: the lack of large-size macrodefects, high growth rate, lower consumption of precursors and effective n-type doping with iodine up to $10^{18} \mathrm{~cm}^{-3}$. The (111)B growth has been enforced by suitable nucleation procedure, based on GaAs substrate annealing in Te-rich atmosphere prior to the growth of CdTe buffer. However, the (111)B layers exhibit some drawbacks which affect the performance of the devices; namely relatively rough surface morphology, $>10^{15} \mathrm{~cm}^{-3}$

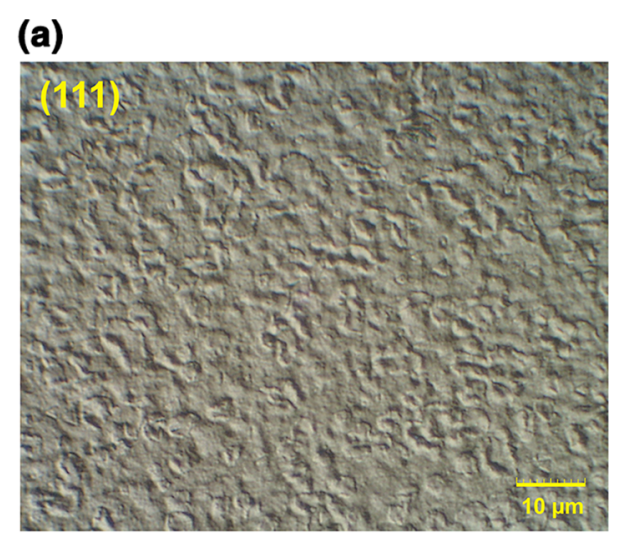

(b)

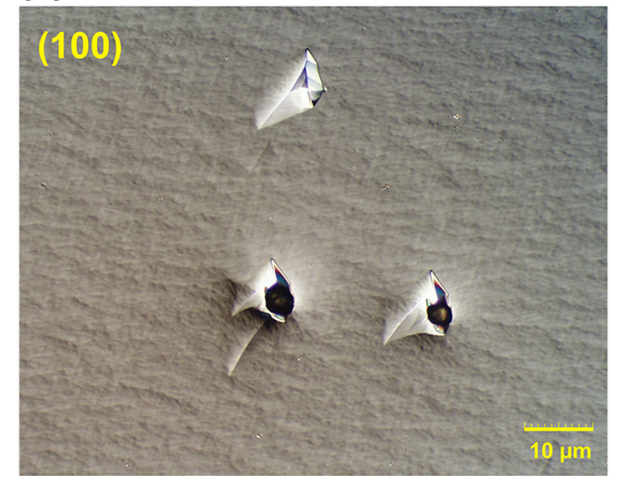

Fig. 1. Surface morphology examples of MOCVD grown (111) $\mathrm{HgCdTe}(\mathrm{a})$ and (100) HgCdTe (b) layers. 


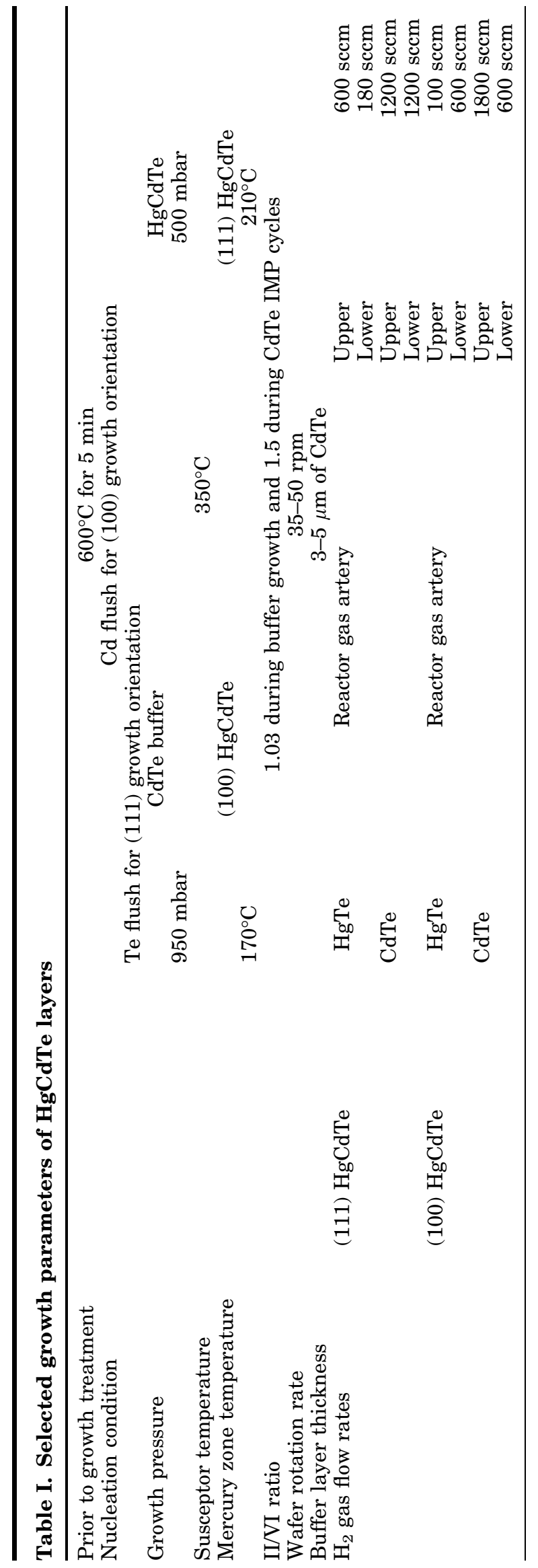




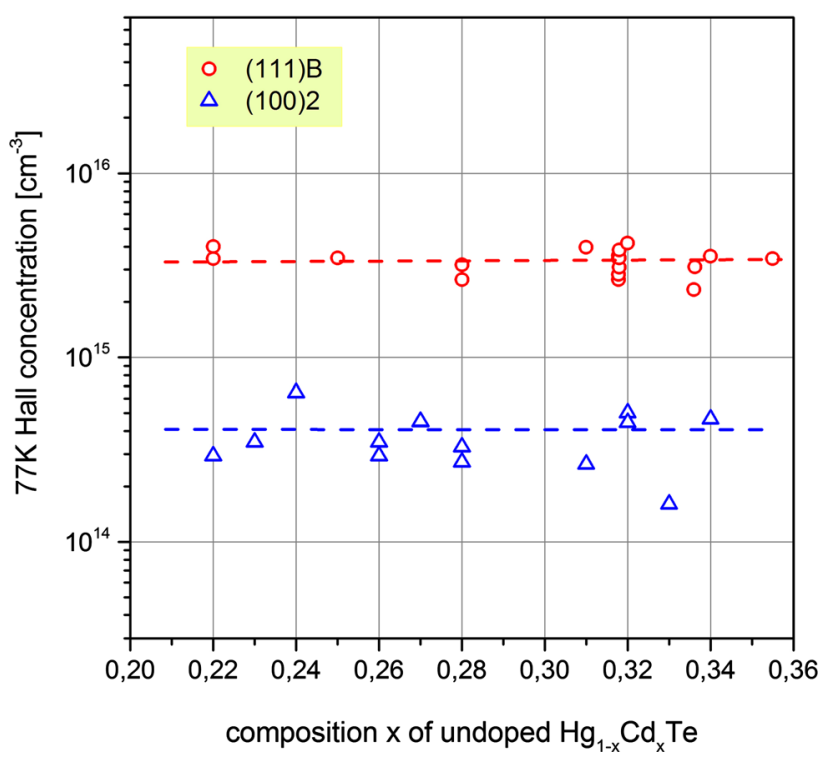

Fig. 2. Hall concentrations of undoped $\mathrm{Hg}_{1-\mathrm{x}} \mathrm{Cd}_{\mathrm{x}} \mathrm{Te}$ layers versus composition $\mathrm{x}$ measured at $77 \mathrm{~K}$.

concentration of residual donor defects and less efficient p-type doping with arsenic.

The MOCVD growth on the (100) orientation was also the subject of our experiments originating and successfully implemented by British research. ${ }^{12}$ The (100) layers exhibit better crystalline quality and related photoelectric properties, but in turn, they show the presence of surface pyramid-shaped macrodefects known as "hillocks". It is very difficult to reduce the density of hillocks to a reasonable level $\left(<10^{3} \mathrm{~cm}^{-2}\right)$. To minimize the macrodefect densities it is essential to optimize the reactor integrity, substrate surface preparation, procedure for removal of the native oxides and numerous nucleation and growth conditions.

The choice of the growth parameters appeared to be quite critical to prevent hillock formation. Cadmium flush during nucleation process provides (100) CdTe growth. ${ }^{13}$

A high potential of MOCVD grown $\mathrm{HgCdTe}$ heterostructures on (211)B was proved by Mitra et al. ${ }^{14}$ This orientation is promising for high operability detector arrays because of the absence of hillock macrodefects. In addition to the surface morphology, the key differences relative to the (100) orientation are in the higher growth rates and the higher iodine incorporation efficiency on the (211)B HgCdTe. However, the drawback of (211)B HgCdTe layers are void defects which originate from the substrate native defects.

The examples of surface morphology of obtained (111)- and (100)-oriented HgCdTe layers are presented in Fig. 1. Neither layer was doped intentionally and they are about $6 \mu \mathrm{m}$ thick. The pictures were obtained using an optical microscope with Nomarski contrast with $\times 1000$ magnification. Both obtained layers are characterized by high uniformity in the composition and the thickness as well

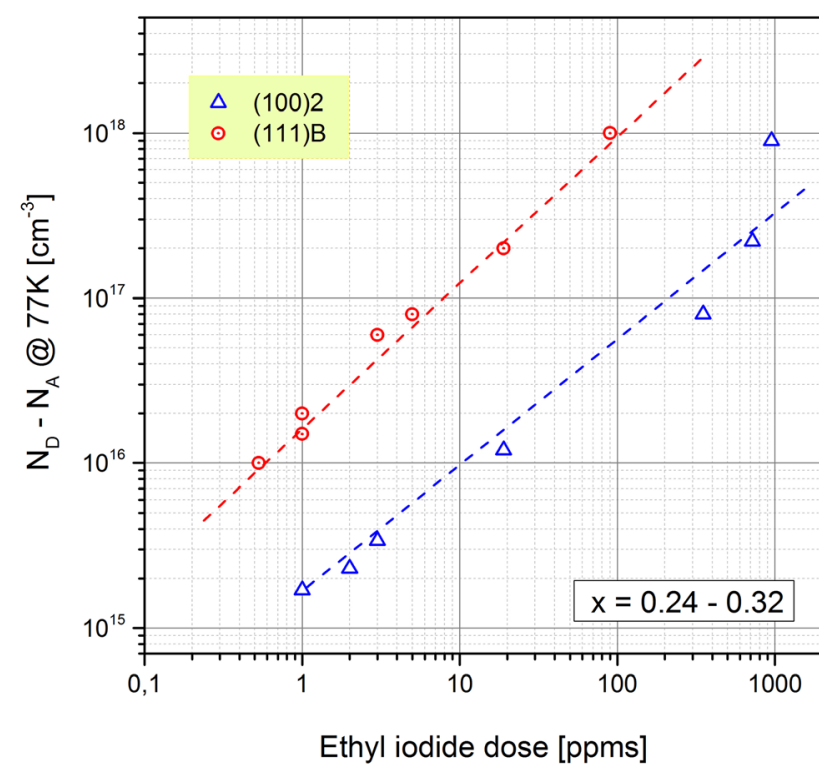

Fig. 3. $77 \mathrm{~K}$ Hall concentration in $\mathrm{HgCdTe}$ as a function of El partial pressure for $(100) 2^{\circ} \rightarrow(110)$ and (111)B.

using Aixtron's gas foil rotation. The standard deviations of the composition, $\mathrm{x}$, and the thickness, $\mathrm{t}$, determined over the whole (2-inch) substrate area are $\sigma_{\mathrm{x}}=0.015$ and $\sigma_{\mathrm{t}}=0.08 \mu \mathrm{m}$, respectively. An area limited to $2 \mathrm{~mm}$ from the wafer edge was excluded from these assessments. Generally, layers with (100) orientation show superior morphology compared to (111) but they are also characterized by hillocks. The height of the hillocks is typically compared to the layer thickness. The hillocks may originate from a small amount of (111) phase appearance during (100) growth. The growth on (100) GaAs substrates, misoriented $2^{\circ}-18^{\circ}$ toward the nearest $<110\rangle$, contributed to a reduction of the twin domain sizes. ${ }^{15,16}$ Rougher surface morphology of (111) HgCdTe layers is probably due to the existence of small amount of (100) phase and threedimensional (111) growth. ${ }^{13}$ Selected and partly optimized growth parameters of HgCdTe layers are listed in Table I.

Figure 2 presents Hall concentrations of undoped $\mathrm{Hg}_{1-\mathrm{x}} \mathrm{Cd}_{\mathrm{x}} \mathrm{Te}$ layers versus composition $\mathrm{x}$ measured at $77 \mathrm{~K}$. Hall concentration of non-intentionally doped $\mathrm{Hg}_{1-\mathrm{x}} \mathrm{Cd}_{\mathrm{x}} \mathrm{Te}$ layers measured at a temperature of $77 \mathrm{~K}$ can be taken to some extent as the purity indicator of a semiconductor. Intrinsic electrons are frozen at $77 \mathrm{~K}$ so Hall concentration reveals the background concentration, which is the result of impurities originating from the substrate, the precursors, the reactor, and the gas delivery system and reflects also the quality of maintenance procedures by the staff. On the other hand, the background concentration may vary with the crystalline quality of the layers. The crystalline imperfections and native defects may act as the generation-recombination centres which influence the electrons and holes concentration. As seen in 


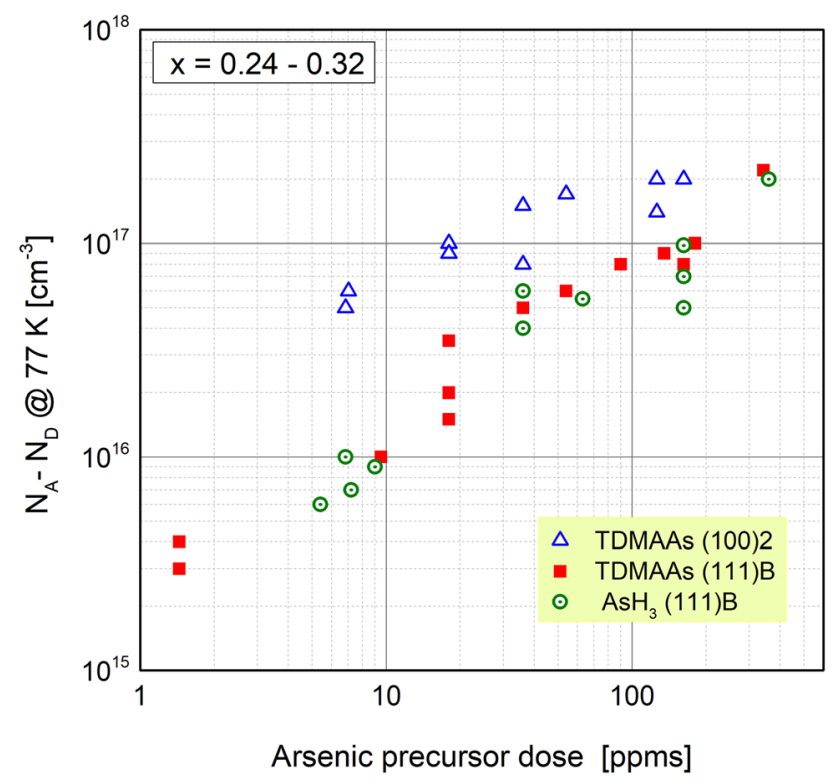

Fig. 4. $77 \mathrm{~K}$ Hall concentration as a function of the arsenic precursor dose in the reactor.

Fig. 2 the background concentration is about $3 \times 10^{15} \mathrm{~cm}^{-3}$ and $4 \times 10^{14} \mathrm{~cm}^{-3}$ for (111)B orientation and (100) $2^{\circ} \rightarrow(110)$ orientation, respectively. An order of magnitude higher background concentration in (111) $\mathrm{HgCdTe}$ is attributable to poorer surface morphology and thus poorer crystalline quality of those layers comparing to (100) $\mathrm{HgCdTe}$.

Figure 3 shows the resultant EI calibration characteristics showing the linear control of donor concentration as a function of the injected EI dose in the reactor for (111) $\mathrm{HgCdTe}$ and (100) $\mathrm{HgCdTe}$. Donor concentrations were taken with Hall measurements at $77 \mathrm{~K}$ using Van der Pauw's method. Iodine incorporation is lower in (100) $\mathrm{HgCdTe}$ of above one order of magnitude in comparison with (111) HgCdTe. Similar observations were reported in Ref. 14. Iodine acts as a donor when it occupies the Te sublattice sites substitutionally. The Te sites on the (111)B surface provide a more stable and favorable adsorption site with three bonds from the underlying group II atoms while the (100) surface provides a weaker adsorption site with two bonds. ${ }^{17}$ Considering the donor, high doping efficiency and high growth rate the (111) $\mathrm{HgCdTe}$ is more favorable for device construction.

Figure 4 presents $77 \mathrm{~K}$ Hall concentration as a function of TDMAAs partial pressure in the reactor for two different crystallographic orientations: (100) $\mathrm{HgCdTe}$ and (111) HgCdTe. The acceptor concentration increases roughly proportionally with As precursor partial pressure up to $150 \mathrm{ppms}$. A higher level of As precursor injection does not result in proportional As-incorporation due to the saturation effect. The arsenic incorporation and doping efficiency strongly depend on crystallographic orientation. Different orientations were obtained by

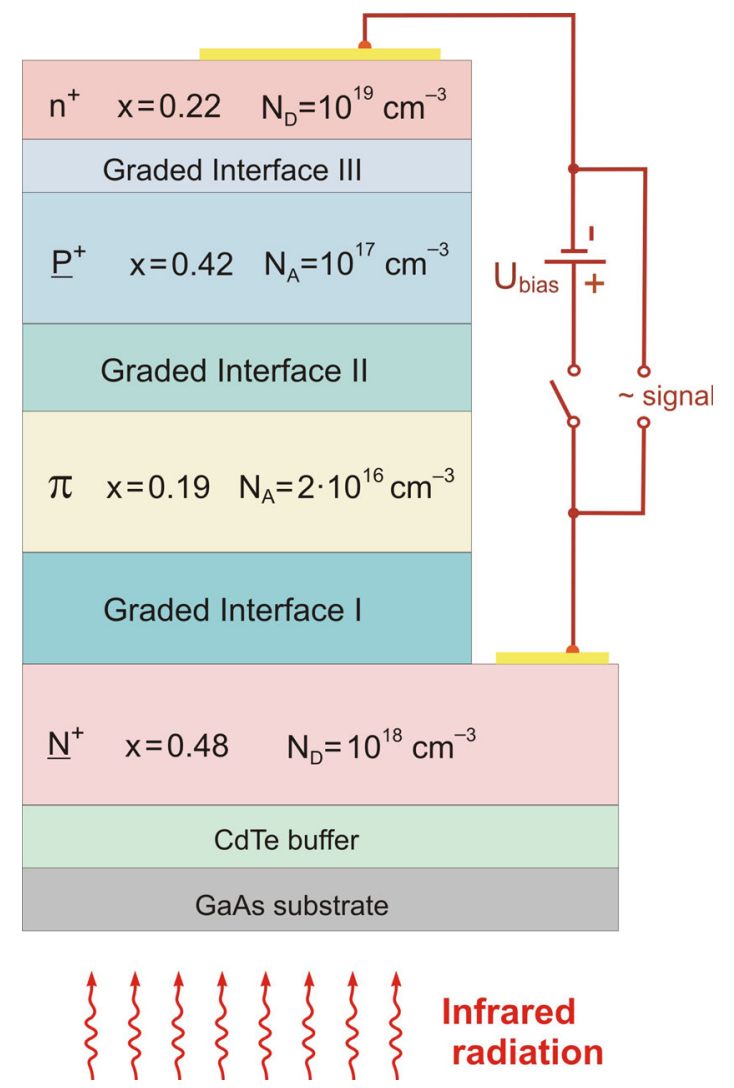

Fig. 5. LWIR HOT HgCdTe structure example.

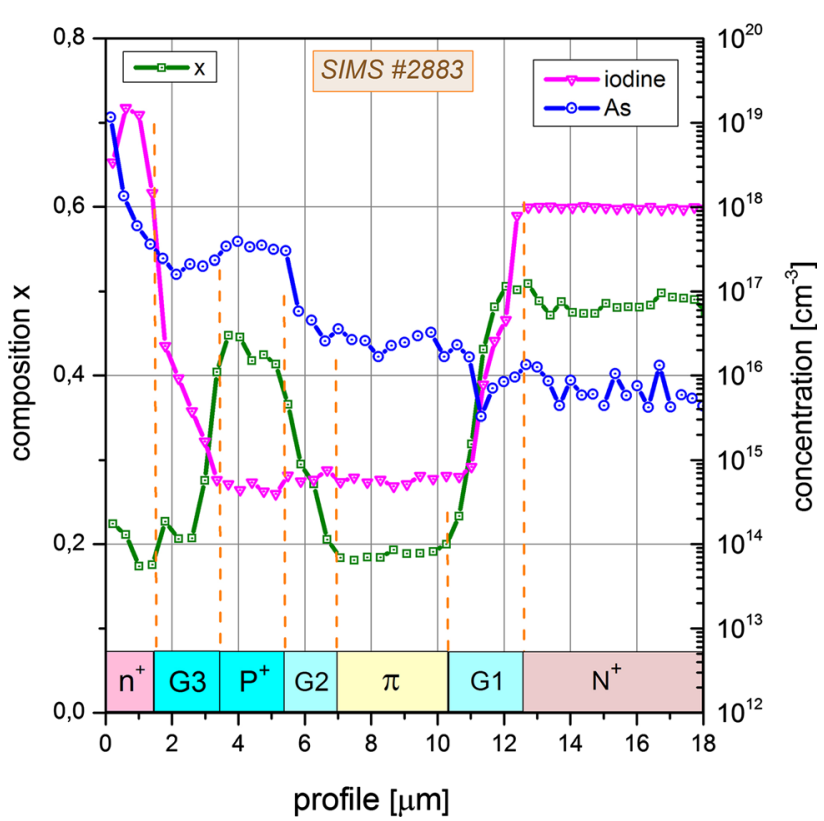

Fig. 6. SIMS profile of $\mathrm{HgCdTe}$ structure type $\mathrm{N}^{+} / \mathrm{G} 1 / \pi / \mathrm{G} 2 / \mathrm{P}^{+} / \mathrm{G} 3 / \mathrm{n}^{+}$

different nucleation conditions. For the same dose of arsenic precursor in the reactor the holes concentration in the (100) $\mathrm{HgCdTe}$ is about one order of magnitude higher than in (111) HgCdTe. This strong polarity dependence can be explained by considering that the two $\mathrm{Cd}$ and As atoms are 
jointly involved in the incorporation process, because the adsorbed species originate from the adduct DMCd-TDMAAs. This complex can break into Cd-As on the surface if proper conditions are realised. In this respect the (100) plane offers the best configuration because this surface has two double-dangling bonds available for both $\mathrm{Cd}$ and $\mathrm{Te}$ (or substituting As) surface atoms. ${ }^{17}$ This mechanism can thus account for the highest doping observed with (100) substrates. Going away from the (100) plane towards (111)A or (111)B leads to reduced As incorporation, and this corresponds with the fact that the relative fraction of (100) terraces decreases at the expense of (111) steps. Arsenic precursor was injected to the reactor during CdTe cycles of the IMP growth process. Introducing TDMAAs during both CdTe and HgTe does not result in an increase of incorporation. These observations are consistent with the results of research published earlier by both Mitra and UK workers. ${ }^{12,14}$ It is worth noting that introducing an As precursor into the reactor accelerates DMCd pyrolysis that causes a considerable shift in $\mathrm{x}$ composition. For example, a 360-ppms dose of TDMAAs shifts x composition from expected 0.3 to 0.4 . Postgrowth annealing at temperatures $\sim 400^{\circ} \mathrm{C}$ did not increase the arsenic activation.

\section{LONG WAVELENGTH PHOTODIODES}

The dark current in LWIR HgCdTe photodiodes is usually determined by Auger generation processes at elevated temperatures, because the low-doped absorber layer becomes intrinsic and the carrier concentration is higher than the doping level. The device structures with a combination of exclusion

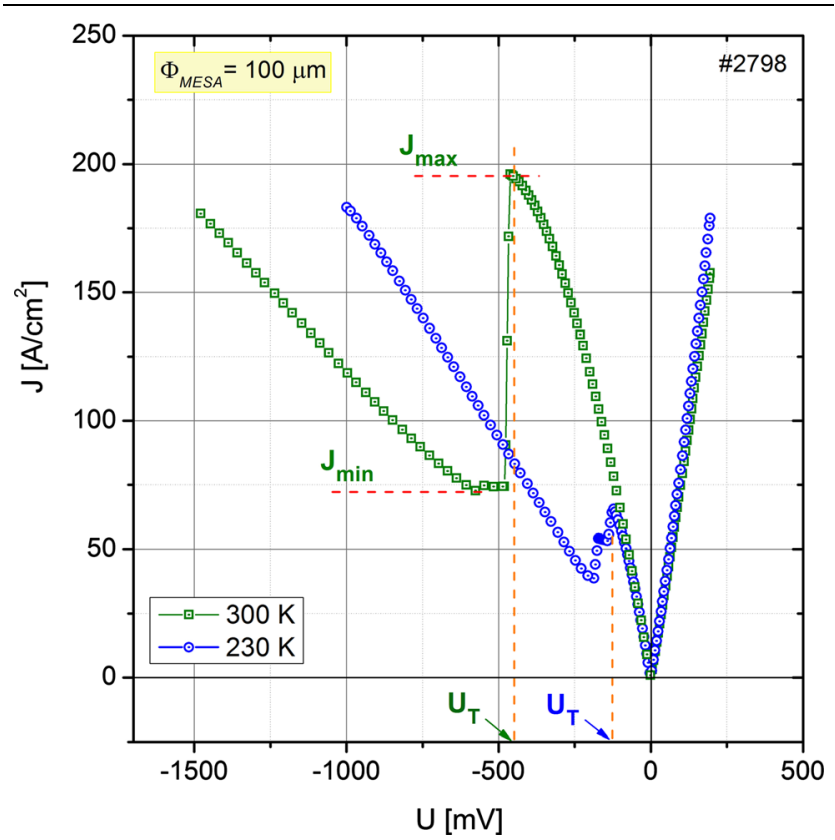

Fig. 7. Dark current density versus bias voltage characteristics of the LWIR HgCdTe diode measured at different temperatures. and extraction junctions in classical $\mathrm{N}^{+} / \pi / \mathrm{P}^{+}$configurations have demonstrated suppression of Auger mechanisms by reducing the absorber carrier density below thermal equilibrium in a reverse bias condition. ${ }^{4}$ Because of interdiffusion processes in HgCdTe material during the growth, the designed $\mathrm{N}^{+} / \pi / \mathrm{P}^{+}$structure takes the form of $\mathrm{N}^{+} / \mathrm{G} / \pi / \mathrm{G} / \mathrm{P}^{+}$ structure, where $\mathrm{G}$ denotes graded interface regions. In order to improve electrical contact properties of metallization to $\mathrm{P}^{+}$layer the structure was upgraded with $\mathrm{p}^{+} / \mathrm{n}^{+}$tunneling junction on the top. Finally, the resulting structure takes the form of $\mathrm{N}^{+} / \mathrm{G} 1 / \pi / \mathrm{G} 2 / \mathrm{P}^{+} / \mathrm{G} 3 / \mathrm{n}^{+} .{ }^{18}$ The example of the architecture of developed devices is presented in Fig. 5. The structure consists of seven different layers which differ in thickness, composition and doping. The thickness of an absorber layer is a compromise between requirements of high absorption and low thermal generation and is about $3.5 \mu \mathrm{m}$. The intrinsic concentration is about $1.6 \times 10^{16} \mathrm{~cm}^{-3}$ in the absorber region for the composition $x=0.196$ at the temperature $T=230 \mathrm{~K}$. There is acceptor doping in the absorber region at the level of $2 \times 10^{16} \mathrm{~cm}^{-3}$ just to overcompensate the intrinsic concentration. As a result, the absorber is lightly $p(\pi)$ type, so the electrons stay as the minority carriers. Highly doped $\mathrm{N}^{+}$and $\mathrm{P}^{+}$layers are thin for low series resistance and their energy gap are much higher than the energy gap of the absorbing region. The whole process of detector architecture designing is predominantly assisted by the numerical

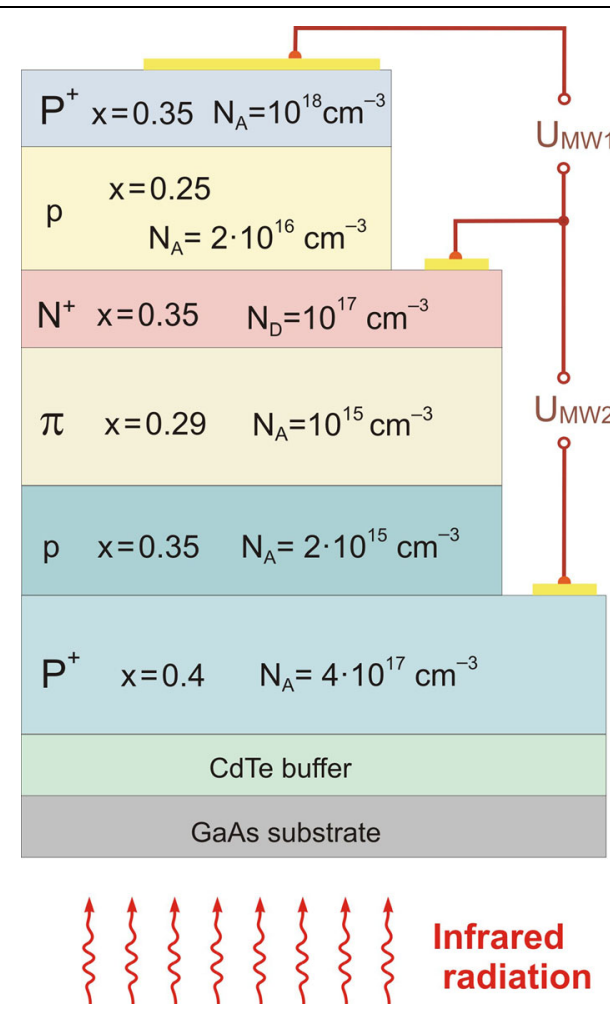

Fig. 8. Two-color $\mathrm{HgCdTe} I R$ detector structure example. 
calculations performed with the APSYS platform (Crosslight Inc.) for example. ${ }^{19}$

Figure 6 shows the secondary ion mass spectrometry (SIMS) profile of $\mathrm{N}^{+} / \mathrm{G} 1 / \pi / \mathrm{G} 2 / \mathrm{P}^{+} / \mathrm{G} 3 / \mathrm{n}^{+}$ heterostructure measured by CAMECA IMS $6 \mathrm{~F}$ set. The solid green line denotes $x$-composition of consecutive $\mathrm{Hg}_{1-\mathrm{x}} \mathrm{Cd}_{\mathrm{x}} \mathrm{Te}$ sublayers according to designed heterostructure shown in Fig. 5. The $x$ composition has been calculated taking into account the measured SIMS points for $\mathrm{Cd}, \mathrm{Hg}$ and Te. We can see quite good convergence between projected and measured values through the whole heterostructure. The diffusion processes during the epitaxial growth cause gradient profile in the interface layers adjacent to the absorber region. For a 135-ppms dose of TDMAAs in deposition of the contact $\mathrm{P}^{+}$-layer, the arsenic concentration is $4 \times 10^{17} \mathrm{~cm}^{-3}$. In spite of the same TDMAAs dose in $\mathrm{p}^{+}(\mathrm{G} 3)$ and $\mathrm{P}^{+}$-regions, the arsenic concentration is slightly smaller in the $\mathrm{p}^{+}(\mathrm{G} 3)$-region than in the $\mathrm{P}^{+}$-region because the doping incorporation only happens in the CdTe growth layers of IMP cycles which are relatively thicker in higher $\mathrm{x} \mathrm{Hg}_{1-\mathrm{x}} \mathrm{Cd}_{\mathrm{x}} \mathrm{Te}$ layers. The gradient slope of the arsenic line between $\mathrm{P}^{+}$and the absorber $\pi$-region seen in Fig. 6 indicates arsenic diffusion from the $\mathrm{P}^{+}$-region to the absorber region, which is dangerous especially in thin, $3-\mu \mathrm{m}$ thick, absorber layers. The iodine profile (the solid pink line with triangles) seen in Fig. 6 indicates expected donor doping levels: $1 \times 10^{19} \mathrm{~cm}^{-3}$ for $\mathrm{n}^{+}$-layer and $1 \times 10^{18} \mathrm{~cm}^{-3}$ for $\mathrm{N}^{+}$-layer with EI doses $90 \mathrm{ppms}$ and $19 \mathrm{ppms}$, respectively. The presence of undesired iodine in the absorber region is at a level of $1 \times 10^{15} \mathrm{~cm}^{-3}$.

The selected parts of the obtained HgCdTe heterostructures were subjected to the standard processing procedure including photolithography,

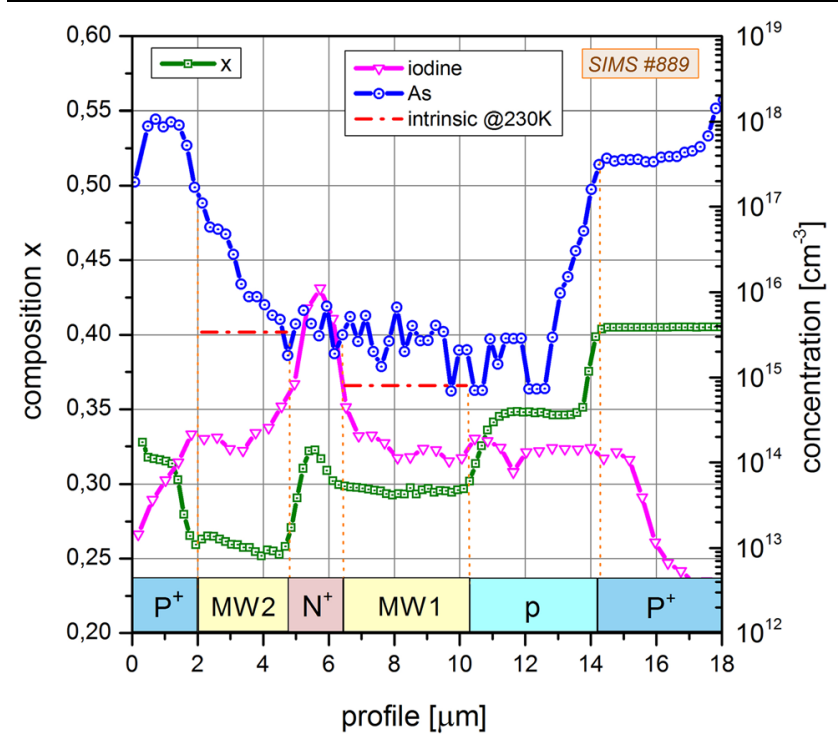

Fig. 9. SIMS profile of two-color $\mathrm{HgCdTe}$ structure type $\mathrm{P}^{+} / \mathrm{MW}^{2} / \mathrm{N}^{+} /$ $\mathrm{MW} 1 / \mathrm{p} / \mathrm{P}^{+}$. chemical etching, metallization and then the test devices were fabricated and measured.

The current-voltage characteristics of LWIR $\mathrm{Hg}_{1}$ ${ }_{\mathrm{x}} \mathrm{Cd}_{\mathrm{x}} \mathrm{Te}$ photodiode measured at different temperatures are presented in Fig. 7. The current-voltage characteristics were measured using the Keithley 2400 sourcemeter. The mesa structure diameter is $100 \mu \mathrm{m}$. A negative differential resistance is seen for both presented characteristics and evidences the suppression of Auger processes due to exclusion and extraction phenomena. The threshold voltage $U_{\mathrm{T}}$ where Auger-suppression commences is about $-170 \mathrm{mV}$ for $T=230 \mathrm{~K}$ and about $-400 \mathrm{mV}$ for $T=300 \mathrm{~K}$. The value of the threshold voltage depends usually on the series resistance and the operating temperature. ${ }^{20}$ The minimal current density value $\left(J_{\min }\right)$ is about $74 \mathrm{~A} / \mathrm{cm}^{2}$ for room temperature characteristic. The differential resistance tends to infinity at both $J_{\min }$ and $J_{\max }$. Biasing the photodiode at the voltage at one of these points provides that the detector resistance is significantly greater than the series resistance even at room temperature and this is more convenient for designing the broadband preamplifiers.

Applying the reverse bias and causing Augersuppression, the current responsivity increases above one order of magnitude. ${ }^{4}$ The time constant of the LWIR photodiodes can be reduced below $200 \mathrm{ps}$ at the reverse bias $U_{\mathrm{b}}<-500 \mathrm{mV}$ at temperature $230 \mathrm{~K}$ as our previous research revealed. ${ }^{18}$

\section{MULTI-COLOR DETECTORS}

Another example of the MOCVD possibilities is deposition of multilayer heterostructures designed for multi-color detectors. In civilian and military programs, environmental sensing and monitoring and target discrimination and identification require simultaneous and independent detection of two or more different spectral ranges in the same pixel of the detector matrix. Figure 8 presents a two-color mid-wavelength HgCdTe IR detector structure type

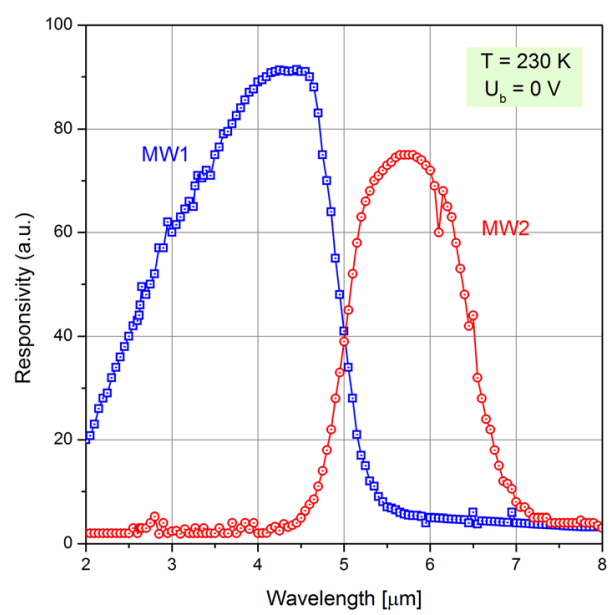

Fig. 10. Spectral response for a dual-band $\mathrm{HgCdTe}$ detector operating in simultaneous mode at $230 \mathrm{~K}$. 


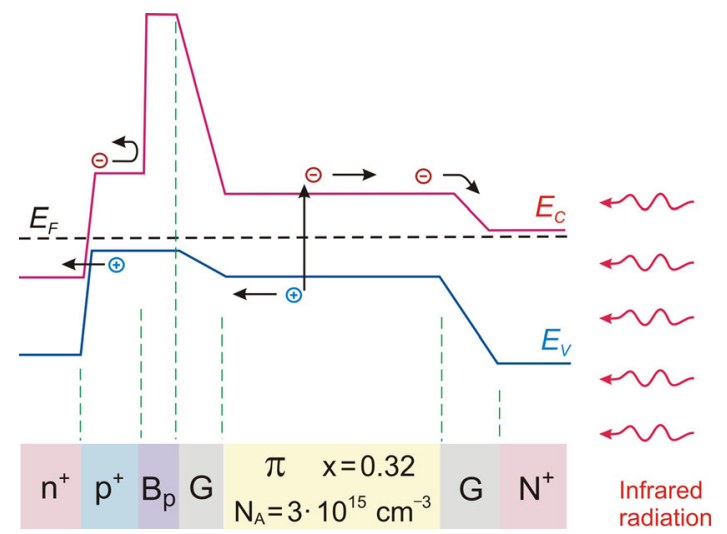

Fig. 11. MWIR $n^{+} p^{+} B_{p} \pi N^{+} H g C d T e$ heterostructure with schematic photodiode band diagram.

$\mathrm{P}^{+} / \mathbf{p} / \mathrm{N}^{+} / \pi / \mathrm{p} / \mathrm{P}^{+}$designed for the simultaneous operation mode. It consists of six layers including two absorbing regions: the lightly doped $\pi$ layer and the medium-doped $\mathbf{p}$ layer with the compositions $x=0.29$ and $x=0.25$, respectively and they act as the MW1 absorber and the MW2 absorber, respectively, at a temperature of $230 \mathrm{~K}$. The $\mathrm{P}^{+}$cladding layers are heavily acceptor doped with composition $\mathrm{x}$ much greater than the absorber's composition. The $\mathrm{N}^{+}$layer is heavily donor doped contact layer which act also as the barrier for the minority carriers preventing them to diffuse into neighboring absorber. The device architecture design is supported by the numerical calculations. ${ }^{21}$

The HgCdTe two-color structures such as that shown in Fig. 8 were grown in an MOCVD system and selected samples were characterized by SIMS measurements. Figure 9 presents the SIMS profile of a two-color HgCdTe structure type $\mathrm{P}^{+} / \mathrm{MW} 2 / \mathrm{N}^{+} /$ $\mathrm{MW} 1 / \mathrm{p} / \mathrm{P}^{+}$. The green squares denote the $x$-composition of consecutive $\mathrm{Hg}_{1-\mathrm{x}} \mathrm{Cd}_{\mathrm{x}} \mathrm{Te}$ sublayers which reflect well designed $x$-values specified in the individual layers depicted in Fig. 8. The exception is the $x$-composition of the $\mathrm{N}^{+}$layer whose value dropped from 0.35 to 0.32 as a result of the diffusion process at the growth temperature equal $350^{\circ} \mathrm{C}$. The pink triangles denote the iodine atoms concentration. The EI dose during the $\mathrm{N}^{+}$layer deposition was 19 ppms which should give approximately $1 \times 10^{18} \mathrm{~cm}^{-3}$ of iodine concentration, but SIMS measurements revealed that this concentration dropped down about two orders of magnitude from what was expected. This drop may result from enhanced iodine diffusion and/or incorrect conversion of SIMS counts to the concentration and/or other unclear reason which resulted in a lower injected iodine dose during the growth than what was assumed. Furthermore, the iodine atoms had spread out through adjacent layers and remained there at an almost constant level $1 \times 10^{14} \mathrm{~cm}^{-3}$, which is undesirable. This conclusion is subjected to some degree of uncertainty, however. It was noted in some SIMS measurements that there were clear

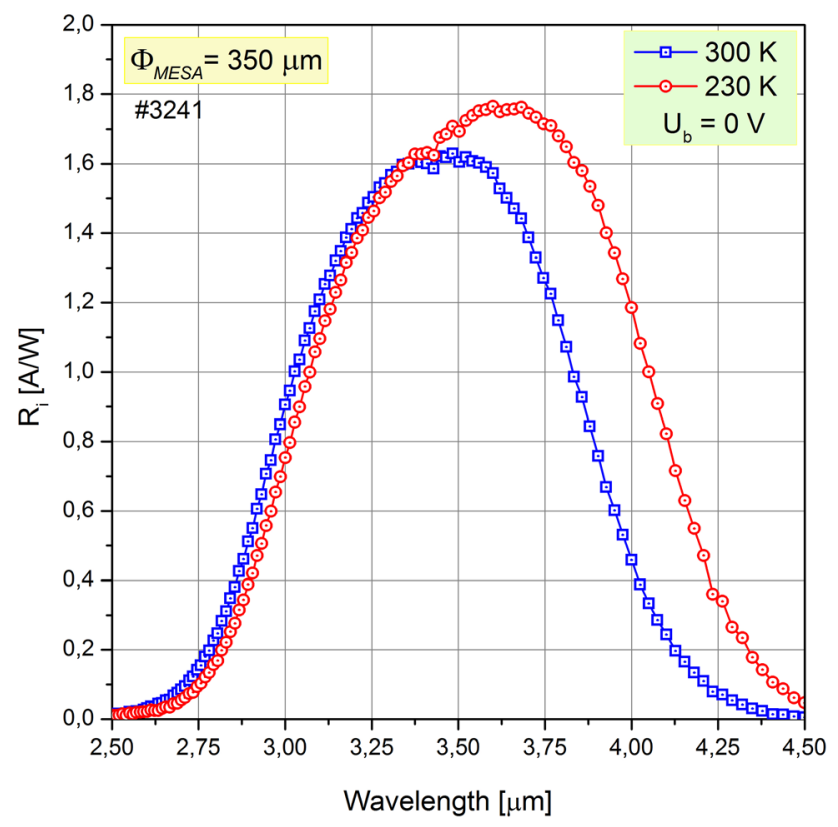

Fig. 12. Current responsivity of the backside-illuminated MWIR $\mathrm{n}^{+} \mathrm{p}^{+} \mathrm{B}_{\mathrm{p}} \pi \mathrm{N}^{+} \mathrm{HgCdTe}$ detector at the temperatures 230 and $300 \mathrm{~K}$.

interferences of iodine counts with tellurium counts (for negative cesium ions), and then residual iodine counts may have incorrectly shifted to just the concentration level of $1 \times 10^{14} \mathrm{~cm}^{-3}$. The blue points represent the arsenic atom concentration. The $\mathrm{P}^{+}$layers were doped with 135 ppms dose of TDMAAs providing high concentration of acceptors and a small resistance of the contacts. We can observe the gradient slope of the arsenic profile between the $\mathrm{P}^{+}$layers and adjacent layers indicates arsenic diffusion from heavily doped $\mathrm{P}^{+}$-region to lightly doped MW1 and MW2 absorbers. The dashdotted red lines denote the intrinsic concentration in the absorbers at the temperature $230 \mathrm{~K}$ and it is located below the arsenic concentration level, so both absorbers are the p-type.

The selected areas of the MOCVD grown HgCdTe heterostructures were subjected to the standard processing procedure and the dual-band devices were prepared and tested.

Figure 10 presents the spectral response for dualband HgCdTe detector operating at the temperature $230 \mathrm{~K}$. The measurements were performed without the bias voltage applying $\left(U_{\mathrm{b}}=0 \mathrm{~V}\right)$. At first, the MW1 pins were connected to the spectrophotometer, and the spectral response characteristic was obtained with the $\lambda_{50 \% \mathrm{CO}}=5 \mu \mathrm{m}$ and next the connections were switched to the MW2 pins and the spectral response was taken with the $\lambda_{50 \% \mathrm{CO}}=$ $6.5 \mu \mathrm{m}$. The MW2 responsivity is of several percentages lower than that of MW1, because the MW2 absorber is too thin and failed to capture all photons. Moreover, there is evident gradient of the composition $\mathrm{x}$ in the MW2, which disorders the 
absorption process. There is also the substantial gradient of the arsenic concentration within the whole area of the MW2 absorber which can contribute to higher recombination of the minority carriers that mutes the signal. The real issue during the development of dual-band detectors are unwanted cross-talk when the signal from one band disturbs the signal form the second band, which was discussed in our previous research.

\section{BARRIER DETECTORS}

Dark current suppressing without impeding the photocurrent flow is the main idea of barrier detectors. ${ }^{22}$ Contrary to the III-V semiconductorbased heterostructures, $\mathrm{HgCdTe}$ material does not exhibit a near zero valance band offset, which is the key issue limiting the performance of barrier detectors nBn type. ${ }^{23}$ However, the proper p-type doping of the barrier layer may reduce the valence bandoffset and increase the offset in the conduction band thus allowing the photogenerated carriers to flow freely in the $\mathrm{HgCdTe}$ material. ${ }^{24}$ As an example of developed $\mathrm{HgCdTe}$ barrier detectors, the $\mathrm{n}^{+} \mathrm{p}^{+} \mathrm{B}_{\mathrm{p}} \pi \mathrm{N}^{+}$ heterostructure for the MWIR range is presented. It consists of a low p-type doped $(\pi)$ absorber, widebandgap heavily doped p-type unipolar barrier $\left(B_{\mathrm{p}}\right)$ and heavily doped contacts. MWIR $\mathrm{n}^{+} \mathrm{p}^{+} \mathrm{B}_{\mathrm{p}} \pi \mathrm{N}^{+}$ $\mathrm{HgCdTe}$ heterostructure with schematic photodiode band diagram is presented in Fig. 11. The unipolar barrier $B_{p}$ blocks the electron injection from $\mathrm{n}^{+}$ contact layer to the absorber region which reduces the dark current. The $\mathrm{p}^{+} / \mathrm{n}^{+}$junction allows the holes to flow easily from the absorber to the left contact by a tunneling mechanism. The introduction of $\mathrm{p}^{+} / \mathrm{n}^{+}$junction allows avoiding technologically difficult $\mathrm{p}$-type contact layer metallization whereas the $\mathrm{n}^{+}$contact layer metallization easy to implement. The absorber composition $x=0.32$ was optimized for absorption in the MWIR range at higher operation temperature $(T>200 \mathrm{~K})$. The p-type absorber doping level of about $N_{\mathrm{A}}=3 \times 10^{15} \mathrm{~cm}^{-3}$ was adjusted in such a way as to compensate the intrinsic concentration which is equal $3.1 \times 10^{14} \mathrm{~cm}^{-3}$ and $2.2 \times 10^{15} \mathrm{~cm}^{-3}$ at the temperatures $230 \mathrm{~K}$ and $300 \mathrm{~K}$, respectively.

Current responsivity $\left(R_{\mathrm{I}}\right)$ of the backside-illuminated MWIR $\mathrm{n}^{+} \mathrm{p}^{+} \mathrm{B}_{\mathrm{p}} \pi \mathrm{N}^{+} \mathrm{HgCdTe}$ detector is presented in Fig. 12. The mesa diameter of the element is about $350 \mu \mathrm{m}$. The measurements were performed at temperatures of $230 \mathrm{~K}$ and $300 \mathrm{~K}$ and without the application of bias voltage $\left(U_{\mathrm{b}}=0 \mathrm{~V}\right)$. $R_{I}$ maximum value is about $1.8 \mathrm{~A} / \mathrm{W}$ and $50 \%$ cut-off wavelength is $4.1 \mu \mathrm{m}$ at a temperature of $230 \mathrm{~K}$. The cut-off wavelength $\lambda_{\mathrm{CO}}$ of measured detector moves to longer wavelengths with decreasing the temperature because the energy gap is narrower there.

\section{CONCLUSIONS}

MOCVD technique with a wide range of composition and donor/acceptor doping is a very convenient tool for the deposition of $\mathrm{HgCdTe}$ epilayers used for uncooled infrared detectors. The unique advantage of MOCVD growth of the HgCdTe heterostructures is that it does not require timeconsuming and unsuitable ex situ post growth annealing in sealed quartz ampoules in the presence of mercury vapors. The detailed study of SIMS profiles of obtained heterostructures revealed diffusion processes of particular elements during the growth. A wide range of different types of infrared detectors operating at HOT conditions has been constructed: photoresistors, non-equilibrium photodiodes, dual-band photodiodes, barriers and multiple detectors.

\section{ACKNOWLEDGMENTS}

This work was supported by the Polish Ministry of Science and Higher Education under PBS 23-897 and the Polish National Centre for Research and Development under the Project POIR.01.01.01-000389/15-00.

\section{CONFLICT OF INTEREST}

The authors declare that they have no conflict of interest.

\section{OPEN ACCESS}

This article is licensed under a Creative Commons Attribution 4.0 International License, which permits use, sharing, adaptation, distribution and reproduction in any medium or format, as long as you give appropriate credit to the original author(s) and the source, provide a link to the Creative Commons licence, and indicate if changes were made. The images or other third party material in this article are included in the article's Creative Commons licence, unless indicated otherwise in a credit line to the material. If material is not included in the article's Creative Commons licence and your intended use is not permitted by statutory regulation or exceeds the permitted use, you will need to obtain permission directly from the copyright holder. To view a copy of this licence, visit http://creativecommons.org/licenses/by/4.0/.

\section{REFERENCES}

1. A. Rogalski, Infrared and Terahertz Detectors, 3rd (Boca Raton: CRC Press, 2019), pp. 221-727.

2. J. Piotrowski and A. Rogalski, High-Operating Temperature Infrared Photodetectors (Bellingham: SPIE Press, 2007).

3. J. Piotrowski and A. Rogalski, Narrow-Gap II-VI Compounds and Electromagnetic Applications, ed. P. Capper (London: Chapman \& Hall, 1997), pp. 506-525. 
4. T. Ashley and C.T. Elliott, Electron. Lett. 21, 451 (1985).

5. J. Piotrowski, W. Gawron, and Z. Djuric, Opt. Eng. 33, 1413 (1994).

6. M. Kopytko, A. Kębłowski, W. Gawron, and W. Pusz, Semicond. Sci. Technol. 31, 035025 (2016).

7. T.J. de Lyon, R.D. Rajavel, J.A. Roth, and J.E. Jensen, Handbook of Infrared Detection and Technologies, ed. M. Henini and M. Razeghi (Oxford: Elsevier, 2002), pp. 309352.

8. C.D. Maxey, P. Capper, and I. Baker, in Metalorganic Vapor Phase Epitaxy (MOVPE): Growth, Materials Properties, and Applications, ed. by S. Irvine, P. Capper (2010). https://doi. org/10.1002/9780470669464.ch6.

9. H. Nishino, S. Murakami, T. Saito, Y. Nishijima, and H. Takigawa, J. Electron. Mater. 24, 533 (1999).

10. J. Tunnicliffe, S.J.C. Irvine, O.D. Dosser, and J.B. Mullin, J. Cryst. Growth 68, 245 (1984).

11. A. Piotrowski and K. Kłos, J. Electr. Mater. 36, 1052 (2007).

12. C.D. Maxey, J.P. Camplin, I.T. Guilfoy, J. Gardner, R.A. Lockett, C.L. Jones, P. Capper, M. Houlton, and N.T. Gordon, Journal of Elec Materi. (2003). http://doi.org/10.1007/ s11664-003-0048-8.

13. W.S. Wang and I. Bhat, Mater. Chem. Phys. (1997). http://d oi.org/10.1016/S0254-0584(97)80290-8.

14. P. Mitra, F.C. Case, and M.B. Reine, Journal of Elec Materi. (1998). http://doi.org/10.1007/s11664-998-0007-5.

15. J.E. Hails, G.J. Russell, A.W. Brinkman, and J. Woods, J. Cryst. Growth (1986). http://doi.org/10.1016/0022-0248(86)9 0576-2.
16. P. Capper, C.D. Maxey, P.A.C. Whiffin, and B.C. Easton, J. Cryst. Growth (1989). http://doi.org/10.1016/0022-0248(89)9 0047-X.

17. L. Svob, I. Cheye, A. Lusson, D. Ballutaud, J.F. Rommeluere, and Y. Marfaing, J. Cryst. Growth (1998). http://d oi.org/10.1016/S0022-0248(98)80096-1.

18. P. Madejczyk, W. Gawron, A. Keblowski, P. Martyniuk, M. Kopytko, W. Pusz, D. Stępień, J. Rutkowski, A. Piotrowski, J. Piotrowski, and A. Rogalski, Opt. Eng. (2017). http://doi. org/10.1117/1.OE.56.8.087103.

19. P. Martyniuk, W. Gawron, J. Pawluczyk, A. Kębłowski, P. Madejczyk, and A. Rogalski, J. Infrared Millim. W. 34, 385 (2015).

20. P.Y. Emelie, S. Velicu, Ch Grein, J.D. Phillips, P.S. Wijewarnasuriya, and N.K. Dhar, J. Electron. Mater. 37, 1362 (2008).

21. M. Kopytko, W. Gawron, A. Kębłowski, D. Stępień, P. Martyniuk, and K. Jóźwikowski, Opt. Quant. Electron. 51, 62 (2019).

22. S. Maimon and G.W. Wicks, Appl. Phys. Lett. 89, 151109 (2006).

23. A.M. Itsuno, J.D. Phillips, and S. Velicu, Appl. Phys. Lett. 100,161102 (2012).

24. M. Kopytko, Infrared Phys. Technol. 64, 47 (2014).

Publisher's Note Springer Nature remains neutral with regard to jurisdictional claims in published maps and institutional affiliations. 